\title{
BMJ Open What is known about the LGBTQ perspective in child welfare services? A scoping review protocol
}

\author{
Jannike Kaasbøll,, ${ }^{1,2}$ Veronika Paulsen ${ }^{3}$
}

To cite: Kaasbøll J, Paulsen V. What is known about the LGBTQ perspective in child welfare services? A scoping review protocol. BMJ Open 2019;9:e030675. doi:10.1136/ bmjopen-2019-030675

- Prepublication history and additional material for this paper are available online. To view these files, please visit the journal online (http://dx.doi org/10.1136/bmjopen-2019030675).

Received 26 March 2019 Revised 13 August 2019 Accepted 21 August 2019

A) Check for updates

(c) Author(s) (or their employer(s)) 2019. Re-use permitted under CC BY-NC. No commercial re-use. See rights and permissions. Published by BMJ.

${ }^{1}$ Department of Mental Health, NTNU, Trondheim, Norway

${ }^{2}$ Department of Health Research, SINTEF, Trondheim, Norway ${ }^{3}$ Department of Social Research, NTNU, Trondheim, Norway

Correspondence to

Dr Jannike Kaasbøll; jannike.kaasboll@ntnu.no

\begin{abstract}
Introduction In previous studies, it is estimated that sexual minorities (eg, lesbian, gay, bisexual, transgender and questioning (LGBTQ) individuals) are overrepresented in the child welfare system. However, the numbers are unclear, and there are limited studies in this field. No systematic review of LGBTQ issues across a broader context (ie, youth, foster parents and service providers) of child welfare services exists. The overall objective of this scoping review is to systematically scope the existing research on LGBTQ issues in the context of child welfare services, including policy, practice, service providers and users' perspectives.
\end{abstract}

Methods and analysis The scoping review framework outlined by the Joanna Briggs Institute (JBI) based on previous work by Arksey and O'Malley and Levac and colleagues will guide this review. In addition, the PRISMA Extension for Scoping Reviews (PRISMA-ScR): Checklist and Explanation will be used throughout the process. We will search electronic databases (PubMed, EMBASE, PsycINFO, Web of Science and Idunn) and grey literature sources to identify studies that are appropriate for inclusion in this review. Using inclusion and exclusion criteria based on the 'Population-Concept-Context' framework, two researchers will independently screen titles, abstracts and full-text articles considered for inclusion. Any qualitative, quantitative and mixed-method study of LGBTQ issues in the child welfare context will be described and synthesised using a thematic synthesis approach.

Ethics and dissemination A scoping review is a secondary analysis of published literature and does not require ethics approval. This scoping review is meant to provide an overview of the existing literature, aiming to expand policy-makers' and practitioners' knowledge of LGBTQ issues in a child welfare context and identify research gaps that can be used as a basis for further research. The results will be disseminated through a peerreviewed publication, a conference presentation and a presentation to the key stakeholders.

\section{INTRODUCTION}

LGBTQ (lesbian, gay, bisexual, transgender and questioning or queer) individuals in the child welfare system have received little attention ${ }^{1}$ Although the focus on LGBTQ individuals has increased in recent years, it is still a very under-researched field internationally.

\section{Strengths and limitations of this study}

This will be the first systematic scoping review of LGBTQ (lesbian, gay, bisexual, transgender and questioning) issues across a broader context (ie, youth, foster parents and service providers) of child welfare services.

- The search strategy will include five electronic databases with peer-reviewed literature and grey literature sources.

- The PRISMA Extension for Scoping Reviews (PRISMA-ScR): Checklist and Explanation will be used throughout the review process.

- Stakeholders, including representatives from users and service providers, will be consulted and engaged throughout the study review process.

This is remarkable, as it has been over 20 years since Sullivan [2, p. 294] pointed at barriers towards effective child welfare services that particularly affect adolescents from sexual minorities. According to Sullivan, these barriers are related to the lack of incorporation of existing research and knowledge into policies and practices, inequalities in the understanding of this group's needs, the lack of suitable foster homes and group homes/ institutions with competence for this group and limited flexibility in such arrangements for older children.

Increasing the awareness, knowledge and skills of social workers and administrators in the child welfare system is of critical importance to effectively meet the needs of LGBTQ youth and foster parents. ${ }^{3}$ However, many social workers and child welfare workers lack the necessary competencies and knowledge to provide LGBTQ individuals with the services to attend to their unique needs. ${ }^{4}$ Mallon and Woronoff ${ }^{5}$ investigated how practitioners, scholars and policymakers treated LGBT (lesbian, gay, bisexual, transgender) youth in foster care. The authors point to a general lack of acknowledging LGBT youth in foster care by traditional child welfare practitioners and policymakers 
and that professionals in the child welfare system are not as progressive towards LGBT issues compared with the general population. Using a national survey of agency members of the Child Welfare League of America (CWLA) with respect to organisational culture and service delivery for lesbian, gay, bisexual, transgender and questioning (LGBTQ) youth, Rosenwald ${ }^{3}$ found that agencies could improve their support for providing an inclusive environment, creating supportive policies and selecting childcare providers regardless of sexual orientation/gender identity. Rosenwald concluded that much work still remains for child welfare agencies to fully address the needs of LGBTQ youth. This underlines the importance of focusing on LGBTQ issues in child welfare services. The current scoping review is meant to provide a basis for identifying research gaps in this field and will also be used as a knowledge base for formulating concrete research questions in our ongoing research project 'LGBTQ-perspective in Child Welfare Services' (2018-2020).

A few recent studies suggest that sexual and gender minorities are overrepresented in child welfare services. ${ }^{15-7}$ As many as $19 \%$ of children and youth in foster care self-identify as LGBTQ ${ }^{6}{ }^{6}$ An estimated one-anda-half to two times as many youths who are LGBTQ are in foster care than are represented in the general population. ${ }^{6}$ Using a national representative sample of children who were referred to child welfare services due to a report of abuse or neglect over a 15-month period in the USA, The Second National Survey of Child and Adolescent Well-Being (NSCAW-II), Fish $e t a l^{8}$ found that sexual minority youth are nearly 2.5 times as likely as heterosexual youth to experience foster care placement and that sexual minority youth are largely overrepresented in child welfare services and out-of-home placement. Dettlaff and colleagues ${ }^{9}$ reported, using the same data (NSCAW-II), that approximately $15.5 \%$ of all systems involved lesbian, gay or bisexual (LGB) youth and that lesbian and bisexual females and LGB youth of colour are both overrepresented within child welfare services. Moreover, LGB youth had an increased risk of adverse mental health outcomes compared with the normal population. Furthermore, based on a cross-sectional study (the California Healthy Kids Survey, USA), Baams et $a l^{10}$ found that youth self-identified as LGBTQ were overrepresented in unstable housing and foster care.

When examining the overrepresentation, it is also important to look at the reasons for young people encountering child welfare services. Forty-four per cent of LGBTQ youth in care reported that their sexual orientation or gender identity were related to their removal from home. ${ }^{11}$ Mostly, LGBTQ youth come into contact with the child welfare system for the same reasons as straight youth. However, LGBTQ youth are excessively overrepresented in cases of parental abandonment and conflict, runaways and truancy. ${ }^{12}$ It can also be differences within the broad group 'LGBTQ', whereas the reasons for child welfare service referrals for many LGBTQ youth more often are related to a child's sexual orientation or gender identity than to their sexual orientation. ${ }^{12}$

Increasing numbers of lesbian women and gay men are accessing fostering and adoption services. Kate Wood ${ }^{13}$ points out that gender and sexuality are still problematic areas of contestation within this context. LGBTQ foster parents and foster-to-adopt families have also received relatively little attention in the published literature. A few studies have examined lesbian women's and gay men's experiences of fostering and/or adopting via foster care. ${ }^{14-20}$ Lavner et $a l^{17}$ call attention to gay men and lesbian women experiencing increased examination and legal obstacles from the child welfare system.

\section{Study rationale}

This scoping review protocol is part of an ongoing project on 'LGBTQ-perspectives in Child Welfare Services' that NTNU Social Research and SINTEF Digital is carrying out with funding from the Norwegian Directorate for Children, Youth and Family Affairs. The aim of the project is to study how the child welfare system encounters children and young people with LGBTQ identity and whether child welfare services are succeeding in meeting the needs of these groups of young people. To obtain an overview of existing national and international research, systematise findings and identify knowledge gaps, we begin by conducting this literature review. The review will provide an important basis for further developing questions and focus areas for the project and will be relevant when developing recommendations for practices. To the best of our knowledge, there is no existing peer-reviewed/published synthesis of the research on LGBTQ issues in a child welfare services setting that incorporates a broad perspective, including LGBTQ youth, LGBTQ foster parents and the perspectives of service providers.

\section{Study objectives}

The overall objective of this scoping review is to systematically scope the existing research on LGBTQ issues in the context of child welfare services, including policy, practice, service providers and users' perspectives, with the following research questions:

1. What is known about the practices of child welfare services towards LGBTQ individuals?

2. What is known about LGBTQ foster parents?

The scoping review will examine the scope and characteristics of the research on the topic 'LGBTQ-perspectives in child welfare services'.

More precisely, the scoping review will:

- Examine the extent (that is, size), range (variety) and nature (characteristics) of the evidence on the topic 'LGBTQ—child welfare services'.

- Summarise the main findings from existing research literature.

- Identify gaps in the literature to guide the planning and commissioning of future research on LGBTQ issues in a child welfare context. 
This work will constitute a first step in a multistep Norwegian research project on 'LGBTQ issues in a child welfare context' and will guide the development and nuances of research questions, interview guides and questionnaires.

\section{METHODS AND ANALYSIS}

A scoping review is considered the most suitable approach, as the method provides an overview of a broad topic. ${ }^{2122}$ Methods will be followed as outlined by the Joanna Briggs Institute (JBI) ${ }^{23}$ based on previous work by Arksey and $\mathrm{O}^{\prime}$ Malley ${ }^{24}$ and Levac and colleagues. ${ }^{25}$ In addition, the PRISMA Extension for Scoping Reviews (PRISMA-ScR): Checklist and Explanation ${ }^{26}$ will be used throughout the review process. The PRIMSA checklist for the present study protocol can be found in the online supplementary material. The scoping review framework ${ }^{23-25}$ consists of six stages: (1) identifying the research question, (2) identifying relevant studies, (3) study selection, (4) charting the data, (5) collating, summarising and reporting results and (6) consultation (optional). These stages will be discussed in further detail below.

\section{Stage 1: identifying the research question}

By consulting the research team and key stakeholders, our overall research questions are: (1) What is known about the practices of child welfare services towards LGBTQ individuals? (2) What is known about LGBTQ foster parents? These questions might be refined, or new ones might be added, as the process of conducting a scoping review is often iterative, requiring a reflexive approach to each stage.

\section{Stage 2: identifying relevant studies}

To identify relevant literature, the search strategy will be underpinned by key inclusion criteria based on the 'Population-Concept-Context (PCC)' framework recommended by the Joanna Briggs Institute for scoping reviews ${ }^{23}$ (table 1). The review will include original research articles (any methods) and review articles, including systematic reviews, meta-analyses, meta-syntheses, narrative reviews, mixed-methods reviews, qualitative reviews and rapid reviews. Grey literature will also be included (research reports). There will be no date limitations. Books and book chapters will be excluded. All settings (outcomes) will be considered.

\section{Search strategy}

Identification of studies relevant to this review will be achieved by searching electronic databases of the published literature and by following the three-step process recommended by the Joanna Briggs Institute. ${ }^{23}$ A preliminary search for previous scoping reviews on the topic or aligning with the topic (The JBI Database of Systematic Reviews and Implementation Reports, Epistemonikos, The Cochrane Database of Systematic Reviews, Campbell Collection and so on) showed that there were no scoping or systematic reviews on the topic. The first step consisted of an initial limited search of at least two online relevant databases. This was undertaken for PubMed (via Ovid) and PsychINFO, using the keywords displayed in box 1 .

The search (27 February 2019) resulted in 287 studies in PubMED and 119 studies in PsychINFO. Potentially relevant text words in the titles and abstracts of the most relevant articles will be used to collect a list of terms and inform a refinement of the terms. The research group will collaborate with a health sciences librarian at the Norwegian University of Science and Technology (NTNU), Faculty of Medicine and Health Science to refine the search.

The second step involves using all identified keywords and index terms and searching all included databases. The following databases will be searched: EMBASE (via Ovid), PsycINFO (via Ovid), Web of Science, PubMed (via Ovid) and the Nordic database Idunn. The search strategy for PubMed can be found in the online supplementary material. In the third and final step, the reference lists of the included studies will be scrutinised and the 'cited-by' function of Google Scholar will be used to search for additional studies. In addition, we will confer with researchers in the field for relevant articles in their country.

As recommended by the Joanna Briggs Institute, a search for a variety of grey literature sources will also be conducted. We will search relevant grey literature databases (eg, Grey Literature Report, OpenGrey, Google

\begin{tabular}{ll}
\hline Table 1 Inclusion and exclusion criteria \\
\hline P-Population & LGBTQ sexual and gender minorities, any age \\
C-Concept & $\begin{array}{l}\text { Child welfare services (eg, in administration and casework, in out-of-home measures such as foster homes } \\
\text { and residential youth care and in-home services such as supervision/guidance to parents and/or young } \\
\text { people/youth/adolescents). Potential outcomes include placement, stability, pathways to care, experiences in } \\
\text { care, practices, attitudes, policy, service access barriers, stigma, support, acceptance, discrimination, service } \\
\text { use and barriers to service access. }\end{array}$ \\
& $\begin{array}{l}\text { The language is limited to English and Scandinavian languages (Norwegian, Swedish and Danish). Research } \\
\text { C-Context } \\
\text { Australia and New Zealand where child welfare services will, to a certain extent, be comparable. }\end{array}$
\end{tabular}

LGBTQ, lesbian, gay, bisexual, transgender and questioning. 


\section{Box 1 Search strategy}

LGBT OR LGBTQ OR lesbian OR gay OR trans* 0 R bisexual ${ }^{*}$ OR queer OR sexual and gender minorities

AND

child welfare OR state custody OR foster care OR foster parent* OR substitute caregiver $\mathrm{OR}$ resource parent

scholar) to identify studies and reports of relevance to this review. Furthermore, we will search the grey literature on the websites of local, national and international organisations and related organisations.

\section{Stage 3: study selection}

References will be imported to EndNote X9 for Windows. Before manual screening commences, duplicates will be removed and articles with missing author and/or title information will be excluded based on EndNote functionality. In the manual process, two independent researchers (JK, VP) will screen the titles of the articles for inclusion based on a priori inclusion criteria. Conflicting decisions will be resolved by discussion. Next, the same process will be repeated based on abstracts. Finally, full texts of potentially relevant studies will be assessed for eligibility by two of the authors (JK, VP). If consensus cannot be achieved, the research team (consisting of seven researchers) will be consulted to make a decision.

\section{Stage 4: charting the data}

In scoping reviews, the data extraction process is referred to as charting the results. ${ }^{23} \mathrm{~A}$ draft charting table/form will be developed at the protocol stage to record the key information of the source, including the following information: author(s); year of publication; origin/country of origin (where the study was published or conducted); aims/purpose of the study; study population and sample size (if applicable); methodology/methods; outcomes and details of these (eg, how measures) (if applicable) and key findings that relate to the scoping review question(s) (eg, placement, stability, pathways to care, experiences in care, practices, attitudes, policy, service access barriers, stigma, support, acceptance, discrimination, service use and barriers to service access). Charting the results can be an iterative process whereby the charting table is continually updated..$^{23}$ Initially, in the data charting process, we will conduct a pilot test of the charting table/form and the information from research-based and non-research-based publications will be collected in separate extraction forms, as recommended by the Joanna Briggs Institute. $^{23}$

\section{Stage 5: collating, summarising and reporting the results}

The checklist for reporting scoping reviews-the 'Preferred Reporting Items for Systematic Reviews and Meta-Analysis: extension for Scoping Reviews (PRISMA-ScR)', the PRISMA-ScR ${ }^{26}$ will be used. As the included studies will vary in study design and methods (ie, qualitative, quantitative and mixed methods), the included studies will be analysed using a thematic synthesis approach. ${ }^{27} 28$ Thematic synthesis has three stages: (1) coding text 'line-by-line'; (2) developing descriptive themes and (3) generating analytical themes. While developing descriptive themes remains close to the primary studies, generating analytical themes represents a stage of interpretation where the reviewers will go beyond the primary studies and generate new interpretive constructs, explanations or hypotheses. ${ }^{27}$ Additionally, we will be able to identify and report gaps in the research in the target field and determine where more in-depth analysis is required in terms of primary research and future systematic reviews. The results will be discussed in the context of current literature, practice and policy.

\section{Stage 6: consultation}

According to the Joanna Briggs Institute, ${ }^{23}$ this step involves a knowledge translation activity and is an important step in scoping reviews. To strengthen the reliability of this study, we will collaborate closely with the rest of the research group in our ongoing research project 'LGBTQ-perspectives in Child Welfare services', which consists of seven researchers (including the authors of this publication) with different perspectives and competences. To validate the findings, we also have a reference group/consultation group consisting of relevant stakeholders in the field of LGBTQ issues and child welfare. We will communicate with this group in three main phases: (1) in the search process-to obtain input on relevant keywords and grey literature; (2) in the analysis process-to ensure knowledge translation and (3) in the end process-to inform and discuss interpretation of the findings. The reference group has representatives from different user organisations, one gay foster parent, child welfare workers and a bachelor's student in child welfare.

\section{Patient and public involvement}

A reference group/consultation group consisting of relevant stakeholders in the field of LGBTQ issues and child welfare will be involved (as described in stage 6 consultation).

\section{Dissemination and ethics}

A scoping review is a secondary analysis of published literature and does not require ethics approval. This work will constitute a first step in a multistep Norwegian research project on 'LGBTQ issues in a child welfare context' and will guide the development and nuances of research questions, interview guides and questionnaires. This scoping review is meant to provide an overview of the existing literature, aiming to expand policy-makers' and practitioners' knowledge of LGBTQ issues in a child welfare context. The results will be disseminated through a peer-reviewed publication, conference presentations and a presentation to the key stakeholders.

Contributors JK and VP conceived of the idea, developed the research question and study methods and contributed meaningfully to the drafting and editing of the final manuscript. 
Funding This work was supported by the Norwegian Directorate for Children, Youth and Family Affairs, SINTEF Digital, Department of Health Research and NTNU Social Research.

Competing interests None declared.

Patient consent for publication Not required.

Provenance and peer review Not commissioned; externally peer reviewed.

Open access This is an open access article distributed in accordance with the Creative Commons Attribution Non Commercial (CC BY-NC 4.0) license, which permits others to distribute, remix, adapt, build upon this work non-commercially, and license their derivative works on different terms, provided the original work is properly cited, appropriate credit is given, any changes made indicated, and the use is non-commercial. See: http://creativecommons.org/licenses/by-nc/4.0/.

\section{REFERENCES}

1. McCormick A, Schmidt K, Terrazas SR. Foster family acceptance: understanding the role of foster family acceptance in the lives of LGBTQ youth. Child Youth Serv Rev 2016;61:69-74.

2. Sullivan TR. Obstacles to effective child welfare service with gay and lesbian youths. Child Welfare 1994;73:291-304.

3. Rosenwald M. A Glimpse Within: An Exploratory Study of Child Welfare Agencies' Practices With LGBTQ Youth. J Gay Lesbian Soc Serv 2009;21:343-56.

4. Mallon GP. Social work practice with lesbian, gay, bisexual and transgender people. Routledge, 2017.

5. Mallon GP, Woronoff R. Busting out of the child welfare closet: lesbian, gay, bisexual, and transgender-affirming approaches to child welfare. Child Welfare 2006;85:115.

6. Wilson BD, Cooper K, Kastanis A, et al. Sexual and gender minority youth in foster care: assessing disproportionality and disparities in Los Angeles. UCLA, 2014.

7. McCormick A, Schmidt K, Terrazas S. LGBTQ youth in the child welfare system: an overview of research, practice, and policy. $J$ Public Child Welf 2017;11:27-39.

8. Fish JN, Baams L, Wojciak AS, et al. Are sexual minority youth overrepresented in foster care, child welfare, and out-of-home placement? findings from nationally representative data. Child Abuse Negl 2019;89:203-11.

9. Dettlaff AJ, Washburn M, Carr LC, et al. Lesbian, gay, and bisexual (LGB) youth within in welfare: prevalence, risk and outcomes. Child Abuse Negl 2018;80:183-93.

10. Baams L, Wilson BDM, Russell ST. LGBTQ youth in unstable housing and foster care. Pediatrics 2019;143:e20174211.

11. Ryan C, Huebner D, Diaz RM, et al. Family rejection as a predictor of negative health outcomes in white and Latino lesbian, gay, and bisexual young adults. Pediatrics 2009;123:346-52.
12. Mallon G. Permanency for LGBTQ youth, 2011: 49-57.

13. Wood K. 'It's all a bit pantomime': an exploratory study of gay and lesbian adopters and foster-carers in England and Wales. Br J Soc Work 2016;46:1708-23.

14. Downs AC, James SE. Gay, lesbian, and bisexual foster parents: strengths and challenges for the child welfare system. Child Welfare 2006;85:281-98.

15. Goldberg A, Moyer AM, Kinkler LA, et al. "When You're Sitting on the Fence, Hope's the Hardest Part": Challenges and Experiences of Heterosexual and Same-Sex Couples Adopting Through the Child Welfare System. Adopt Q 2012;15:288-315.

16. Goldberg AE, Kinkler LA, Moyer AM, et al. Intimate relationship challenges in early parenthood among lesbian, gay, and heterosexual couples adopting via the child welfare system. Prof Psychol 2014;45:221-30.

17. Lavner JA, Waterman J, Peplau LA. Parent adjustment over time in gay, lesbian, and heterosexual parent families adopting from foster care. Am J Orthopsychiatry 2014;84:46-53.

18. Hicks S. Genealogy's desire: practices of kinship amongst Lesbian and gay Foster-Carers and Adopters. Br J Soc Work 2005;36:761-76.

19. Matthews JD, Cramer EP. Envisaging the adoption process to strengthen gay- and lesbian-headed families: recommendations for adoption professionals. Child Welfare 2006;85:317-40.

20. Mallon GP. Assessing lesbian and gay prospective foster and adoptive families: a focus on the home study process. Child Welfare 2007;86:67.

21. Munn Z, Peters MDJ, Stern C, et al. Systematic review or scoping review? Guidance for authors when choosing between a systematic or scoping review approach. BMC Med Res Methodol 2018;18:143.

22. Peterson J, Pearce PF, Ferguson LA, et al. Understanding scoping reviews: definition, purpose, and process. J Am Assoc Nurse Pract 2017;29:12-16.

23. Institute JB. The Joanna Briggs Institute Reviewers' Manual 2015 Methodology for JBI Scoping Reviews. Australia: The Joanna Briggs Institute, 2015.

24. Arksey H, O'Malley L. Scoping studies: towards a methodological framework. Int J Soc Res Methodol 2005;8:19-32.

25. Levac D, Colquhoun H, O'Brien KK. Scoping studies: advancing the methodology. Implement Sci 2010;5.

26. Tricco AC, Lillie E, Zarin W, et al. PRISMA extension for scoping reviews (PRISMA-ScR): checklist and explanation. Ann Intern Med 2018;169:467-73.

27. Thomas J, Harden A. Methods for the thematic synthesis of qualitative research in systematic reviews. BMC Med Res Methodol 2008;8:45.

28. Thomas J, Harden A, Oakley A, et al. Integrating qualitative research with trials in systematic reviews. BMJ 2004;328:1010-2. 